\title{
Functional interactions of the cystine/glutamate antiporter, CD44V and MUC1-C oncoprotein in triple-negative breast cancer cells
}

\author{
Masanori Hasegawa ${ }^{1}$, Hidekazu Takahashi ${ }^{1,3}$, Hasan Rajabi ${ }^{1}$, Maroof Alam ${ }^{1}$, Yozo \\ Suzuki,1, Li Yin ${ }^{1}$, Ashujit Tagde ${ }^{1}$, Takahiro Maedaํ, Masayuki Hiraki ${ }^{1}$, Vikas P. \\ Sukhatme ${ }^{2}$ and Donald Kufe ${ }^{1}$ \\ ${ }^{1}$ Dana-Farber Cancer Institute, Harvard Medical School, Boston, MA, USA \\ 2 Beth Israel Deaconess Medical Center, Harvard Medical School, Boston, MA, USA \\ ${ }^{3}$ Department of Gastrointestinal Surgery, Graduate School of Medicine, Osaka University, Suita City, Osaka, Japan \\ ${ }^{4}$ Department of Gastroenterological Surgery, Osaka Police Hospital, Osaka City, Osaka, Japan \\ Correspondence to: Donald Kufe, email: donald_kufe@dfci.harvard.edu \\ Keywords: XCT, MUC1-C, CD44v, epigenetics, ferroptosis \\ Received: December 11,2015 Accepted: January 26, $2016 \quad$ Published: February 22, 2016
}

\section{ABSTRACT}

The $\mathrm{XCT}$ light chain of the cystine/glutamate transporter (system $\mathrm{X}_{\mathrm{c}}{ }^{-}$) is of importance for the survival of triple-negative breast cancer (TNBC) cells. The MUC1-C transmembrane oncoprotein is aberrantly overexpressed in TNBC and, like XCT, has been linked to maintaining glutathione (GSH) levels and redox balance. However, there is no known interaction between MUC1-C and XCT. Here we show that silencing MUC1-C is associated with decreases in XCT expression in TNBC cells. The results demonstrate that MUC1-C forms a complex with XCT and the CD44 variant (CD44v), which interacts with XCT and thereby controls GSH levels. MUC1-C binds directly with CD44v and in turn promotes stability of XCT in the cell membrane. The interaction between MUC1-C and XCT is further supported by the demonstration that targeting XCT with silencing or the inhibitor sulfasalazine suppresses MUC1 gene transcription by increasing histone and DNA methylation on the MUC1 promoter. In terms of the functional significance of the MUC1-C/XCT interaction, we show that MUC1-C protects against treatment with erastin, an inhibitor of $X_{c}{ }^{-}$and inducer of ferroptosis, a form of non-apoptotic cell death. These findings indicate that targeting this novel MUC1-C/ XCT pathway could represent a potential therapeutic approach for promoting TNBC cell death.

\section{INTRODUCTION}

The system $\mathrm{X}_{\mathrm{C}}^{-}$cystine/glutamate antiporter is a member of the family of heterodimeric amino acid transporters. System $X_{C}^{-}$is composed of a light chain, designated $\mathrm{xCT}$, and the $4 \mathrm{~F} 2$ heavy chain that traffics the heterodimer to the cell membrane [1]. xCT functions as a major transporter for the uptake of cystine in exchange for glutamate. xCT-mediated uptake of cystine and its intracellular reduction to cysteine, constitute a redox couple that maintains balance between extracellular cystine and cysteine [1]. Additionally, intracellular cysteine is the rate-limiting precursor for the synthesis of glutathione (GSH), a tripeptide consisting of glutamate, cysteine and glycine, that plays a critical role in the cellular defense against oxidative stress [2]. As a result, cells from $\mathrm{xCT}$-deficient mice exhibit decreases in cysteine and GSH levels [3]. XCT is expressed by diverse cancer cells and contributes to their growth, survival and resistance to anti-cancer agents, at least in part, by maintaining GSH levels and thereby redox balance [4-6]. The CD44 variant (CD44v), a marker of cancer stem-like cells (CSCs), stabilizes $\mathrm{xCT}$ at the cell membrane and thus enhances cystine uptake for GSH synthesis in gastrointestinal cancer cells [7]. Moreover, ablation of CD44 suppresses $\mathrm{xCT}$ function, induces oxidative stress and inhibits gastric tumor growth in transgenic mice [7]. Other work has shown that $\mathrm{xCT}$ is expressed in triple-negative breast cancers (TNBC) and contributes to glutamine dependency [8]. In this way, conversion of glutamine to glutamate is 
needed for $\mathrm{xCT}$-mediated uptake of cystine. Inhibition of $\mathrm{xCT}$ in TNBC cells with the clinically approved antiinflammatory agent sulfasalazine (SASP) decreases cystine uptake, GSH production and tumor growth [8]. These and other findings that chemotherapy induces $\mathrm{xCT}$ expression in TNBC cells [9] have supported the notion that $\mathrm{xCT}$ represents a potential target for cancer cell-specific GSH depletion and, in turn, sensitization to treatment with anti-cancer agents that disrupt redox balance $[5,10,11]$.

The mucin 1 (MUC1) transmembrane glycoprotein is aberrantly overexpressed in most breast cancers, including about $90 \%$ of TNBCs $[12,13]$. MUC1 consists of two subunits that form a heterodimeric complex at the cell membrane [12]. The MUC1 N-terminal subunit (MUC1-N) contains glycosylated tandem repeats that are characteristic of the mucin family. The oncogenic MUC1 C-terminal subunit (MUC1-C) consists of a 58 amino acid (aa) extracellular domain, a 28 aa transmembrane region and a 72 aa cytoplasmic tail [12]. Overexpression of the MUC1-N/MUC1-C complex has been associated with amplification of the MUC1 gene at chromosome 1q21 in about $40 \%$ of breast cancers $[14,15]$. In addition, the MUC1-C subunit forms auto-inductive interactions with the NF- $\kappa$ B p65 and STAT1/3 transcription factors that confer activation of the $M U C 1$ promoter and thereby MUC1-C expression in breast cancer cells [16-18]. Studies in breast cancer cells have further supported epigenetic regulation of $M U C 1$ promoter activation through histone modification and DNA methylation [19]. With loss of apical-basal polarity as found in carcinoma cells, MUC1-C is expressed over the entire cell membrane where it interacts with receptor tyrosine kinases, such as EGFR and HER2, and promotes their activation [20, 21]. The MUC1-C cytoplasmic domain is an intrinsically disordered protein that interacts with multiple effectors, such as PI3K, NF- $\mathrm{B}$ p 65 and $\beta$-catenin, which have been associated with transformation $[12,22]$. In addition and like $\mathrm{xCT}$, MUC1-C has been linked to the regulation of GSH and maintenance of intracellular redox balance $[12,23]$. The overexpression of MUC1-C is sufficient to induce anchorage-independent growth and tumorigenicity, supporting its function as an oncoprotein [12]. Other studies have shown that MUC1-C confers self-renewal of breast cancer cells [24]. Thus, targeting MUC1-C with genetic approaches or treatment with inhibitors blocks the capacity of breast cancer cells, including those of the TNBC subtype, to form mammospheres and tumors in mice [24]. These findings have supported the notion that MUC1-C contributes to TNBC cell survival.

The present studies have investigated the potential relationship between MUC1-C and $\mathrm{xCT}$ based on the findings that both are of importance for redox balance and self-renewal of TNBC cells $[8,24]$. Our results demonstrate that MUC1-C associates with the $\mathrm{xCT} /$ CD44v complex in TNBC cells and stabilizes $\mathrm{xCT}$. In turn, we show that targeting $\mathrm{xCT}$ suppresses MUC1-C expression by promoting epigenetic modifications of the $M U C 1$ promoter. Our findings provide further support for a model in which MUC1-C and XCT function in a pathway that regulates ferroptosis and thereby survival of TNBC cells.

\section{RESULTS}

\section{MUC1-C interacts with $\mathrm{xCT}$}

MUC1-C and the $\mathrm{xCT}$ antiporter are both aberrantly expressed in TNBC cells [8, 13]; however, there is no known interaction between these two cell membrane proteins. Studies performed with MDA-MB-468 TNBC cells demonstrated that MUC1-C coprecipitates with $\mathrm{xCT}$ (Figure 1A, left). The detection of MUC1-C/xCT complexes was confirmed when anti-xCT precipitates were analyzed by immunoblotting with anti-MUC1-C (Figure 1A, right). Similar results obtained with BT20 TNBC cells provided further support that MUC1-C associates with $\mathrm{xCT}$ (Figure $1 \mathrm{~B}$, left and right). To assess the functional significance of the MUC1-C/ $\mathrm{xCT}$ interaction, we generated TNBC cells with tetracycline inducible expression of a MUC1 shRNA (tet-MUC1shRNA) or a control shRNA (tet-CshRNA). Treatment of MDA-MB-468/tet-MUC1shRNA cells with DOX for $48 \mathrm{~h}$ was associated with suppression of membrane-associated MUC1-C (Figure 1C) and total cellular MUC1-C (Supplementary Figure S1A). Notably, doxycycline (DOX)-induced MUC1-C suppression in MDA-MB-468/tet-MUC1shRNA cells was also associated with decreases in $\mathrm{xCT}$ levels (Figure $1 \mathrm{C}$ and Supplementary Figure S1A). By contrast, DOX had no effect on MUC1-C or $\mathrm{xCT}$ expression in the control MDA-MB-468/tet-CshRNA cells (Supplementary Figure S1B). Similar results were obtained with DOX-treated BT20/tet-MUC1shRNA and BT-20/tet-CshRNA cells (Figure 1D and Supplementary Figures S1C and S1D), indicating that silencing MUC1-C downregulates xCT levels. xCT functions in the transmembrane exchange of extracellular cysteine and intracellular glutamate. In concert with the downregulation of xCT, DOX treatment of MDA-MB-468/ tet-MUC1shRNA and BT-20/tet-MUC1shRNA cells was associated with significant increases in intracellular glutamate (Figure 1E, left and right). These findings provided support for the notion that targeting MUC1-C promotes the downregulation of $\mathrm{xCT}$ expression.

\section{MUC1-C interacts with the $x C T / C D 44$ complex}

$\mathrm{xCT}$ forms a complex with the CD44 variant $(\mathrm{CD} 44 \mathrm{v})$ at the cell membrane [7]. Analysis of MDAMB-468 and BT-20 cells demonstrated that, like $\mathrm{xCT}$, 
MUC1-C associates with CD44v (Figure 2A and Supplementary Figure S2). To define the nature of the association between MUC1-C and CD44v, we performed binding studies with the MUC1-C cytoplasmic domain (MUC1-CD) and the CD44 intracellular domain (CD44ICD). The experiments revealed a direct interaction between MUC1-CD and CD44-ICD (Figure 2B). Moreover, using deletion mutants, we found that CD44ICD binds to MUC1-CD(1-45), but not to MUC1$\mathrm{CD}$ (20-72), indicating that MUC1-CD amino acids 1-19 confer the interaction with CD44-ICD (Figure 2B). In addition, binding studies with CD44-ICD fragments demonstrated that MUC1-CD interacts with CD44-ICD amino acids 34-44 (Figure 2C). The CD44v9 region is of importance for the interaction between CD44v and $\mathrm{xCT}$ [7]. We found that the CD44v9 variant is highly expressed in MDA-MB-468 and BT-20 TNBC cells as compared to that in luminal ZR-75-1 and MCF-7 breast cancer cells (Figure 2D, left and right). Accordingly, we asked if CD44v9 contributes to the interaction between MUC1-C and $\mathrm{xCT}$. In support of this notion, transient overexpression of CD44v8-10 in 293T/MUC1-C cells was associated with increases in MUC1-C/xCT complexes as compared to that in 293T/vector cells (Figure 2E). These findings supported a model in which MUC1-C associates with the $\mathrm{xCT} / \mathrm{CD} 44 \mathrm{v}$ complex, at least in part, through a direct interaction with CD44v.

\section{MUC1-C promotes XCT stabilization}

CD44v stabilizes $\mathrm{xCT}$ at the cell membrane and thereby regulates redox balance in cancer cells [7]. To determine whether the interaction of MUC1-C with $\mathrm{xCT} / \mathrm{CD} 44 \mathrm{v}$ affects $\mathrm{xCT}$ expression, we first stably overexpressed MUC1-C in MUC1-null 293T cells (Figure $3 \mathrm{~A}$, left). As found in TNBC cells, MUC1-C forms complexes with $\mathrm{xCT}$ (Figure 3A, right). We also found that MUC1-C expression is associated with increases in $\mathrm{xCT}$ levels in the membrane fraction (Figure 3B, left) and in total cell lysates (Figure 3B, right). Analysis of xCT stability in the presence of cyclohexamide (CHX) further demonstrated that MUC1-C expression is associated with an increase in $\mathrm{xCT}$ half-life (Figures $3 \mathrm{C}$ and 3D). In concert with these results, silencing MUC1-C in MDAMB-468 (Figures 3E and 3F) and BT-20 (Supplementary Figures S3A and S3B) cells was associated with a decrease in $\mathrm{xCT}$ stability. These results thus provided support for involvement of MUC1-C in the stabilization of $\mathrm{xCT}$.
A. MDA-MB-468

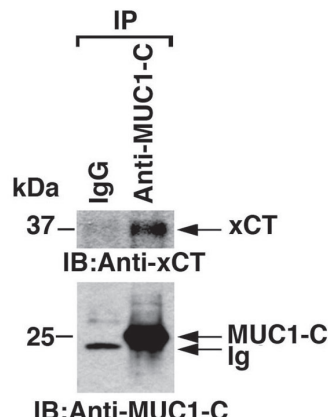

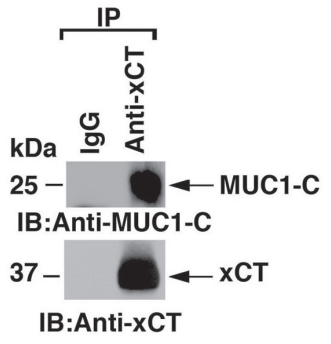

IB:Anti-xCT
B. BT-20
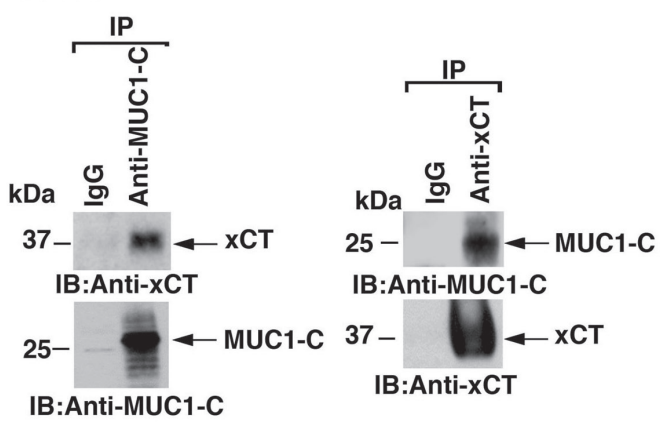

E.

F. MDA-MB-468/tet-MUC1shRNA

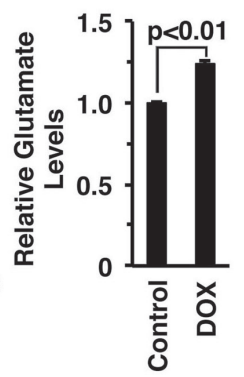

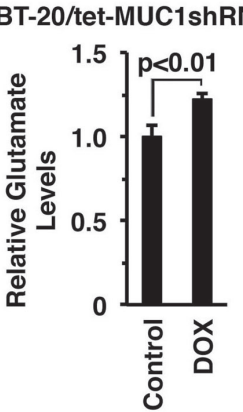

Figure 1: Downregulation of MUC1-C decreases xCT levels. A. and B. Lysates from MDA-MB-468 (A) or BT-20 (B) cells were precipitated with anti-MUC1-C, anti-xCT or a control IgG. The precipitates were immunoblotted with the indicated antibodies. C. and D. MDA-MB-468/tet-MUC1shRNA (C) and BT-20/tet-MUC1shRNA (D) cells were treated with $200 \mathrm{ng} / \mathrm{ml}$ DOX for the indicated times. Membrane fractions were immunoblotted with the indicated antibodies. E. and F. Intracellular glutamate levels were determined in MDA-MB-468/tet-MUC1shRNA (E) and BT-20/tet-MUC1shRNA (F) cells cultured in the presence of $200 \mathrm{ng} / \mathrm{ml} \mathrm{DOX} \mathrm{for} 72 \mathrm{~h}$. The results (mean $\pm \mathrm{SD}$ of 3 determinations) are expressed as relative glutamate levels as compared with that obtained from cells without DOX treatment (assigned a value of 1). 
A. MDA-MB-468

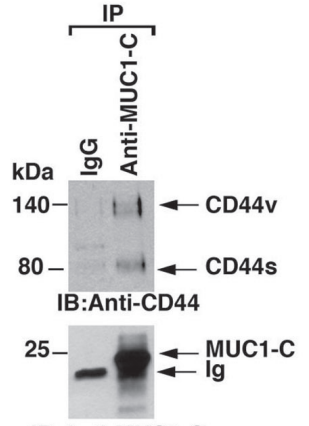

IB:Anti-MUC1-C
B.

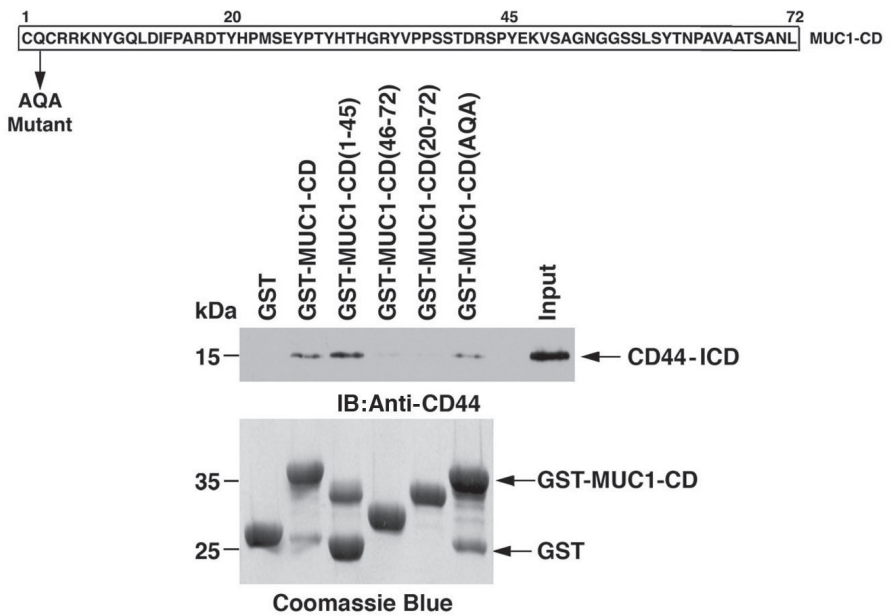

C.
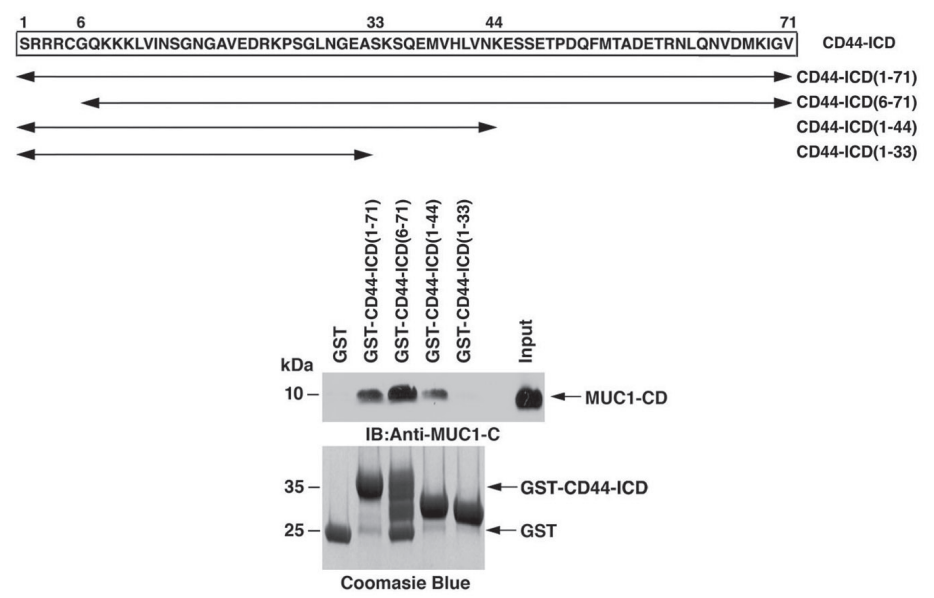

D.

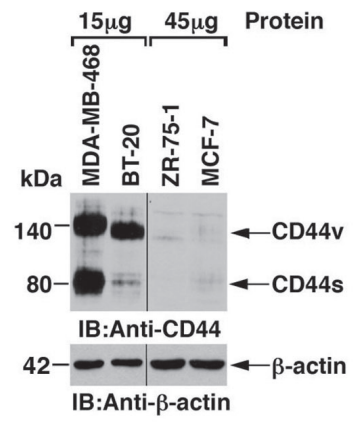

E. 293T

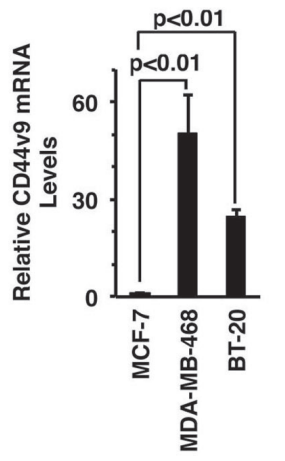

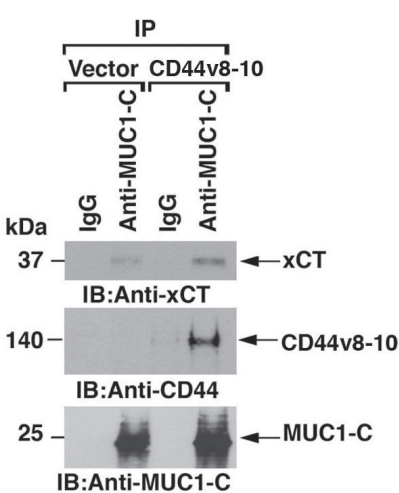

Figure 2: MUC1-C interacts with CD44v. A. Lysates from MDA-MB-468 cells were precipitated with anti-MUC1-C or a control IgG. The precipitates were immunoblotted with the indicated antibodies. B. Amino acid sequence of the MUC1 cytoplasmic domain (MUC1-CD). GST, GST-MUC1-CD or the indicated GST-MUC1-CD fragments were incubated with His-CD44-ICD. The adsorbates were immunoblotted with anti-CD44. Input of the GST proteins was assessed by Coomassie blue staining. C. Amino acid sequence of the CD44-ICD. GST, GST-CD44-ICD or the indicated GST-CD44-ICD fragments were incubated with MUC1-CD. The adsorbates were immunoblotted with anti-MUC1-C. Input of the GST proteins was assessed by Coomassie blue staining. D. Lysates (15 and $45 \mu \mathrm{g})$ from MDA-MB-468, BT-20, ZR-75-1 and MCF-7 cells were immunoblotted with the indicated antibodies (left). CD44v9 mRNA levels in the indicated cells were determined by qRT-PCR (right). The results (mean \pm SD of 4 determinations) are expressed as relative CD $44 \mathrm{v} 9 \mathrm{mRNA}$ levels as compared with that obtained for MCF-7 cells (assigned a value of 1). E. Lysates from 293T/MUC1-C cells transfected with an empty vector or one expressing CD44v8-10 were precipitated with anti-MUC1-C or a control IgG. The precipitates were immunoblotted with the indicated antibodies. 


\section{Targeting xCT decreases MUC1-C expression}

In experiments assessing the interaction between MUC1-C and $\mathrm{xCT}$, we treated MDA-MB-468 cells with the $\mathrm{xCT}$ inhibitor SASP. Surprisingly, SASP treatment was associated with marked suppression of MUC1-C mRNA and protein (Figures 4A, left and right). Significant effects of SASP on MUC1-C expression were also observed in BT-20 cells (Figure 4B, left and right). To provide further evidence that $\mathrm{xCT}$ regulates $\mathrm{MUC1}$ expression, we stably silenced $\mathrm{xCT}$ and found associated decreases in MUC1 mRNA and protein in MDA-MB-468 (Figure 4C) and BT20 (Figure 4D) cells. Similar results were obtained with MDA-MB-468 (Supplementary Figure S4A) and BT-

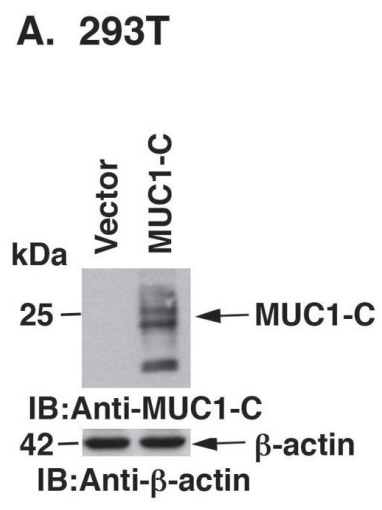

B. 293T

Membrane

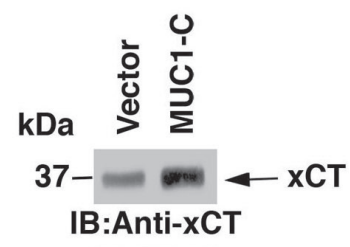

$100-\square$ Na,K ATPase

IB:Anti-Na,K ATPase

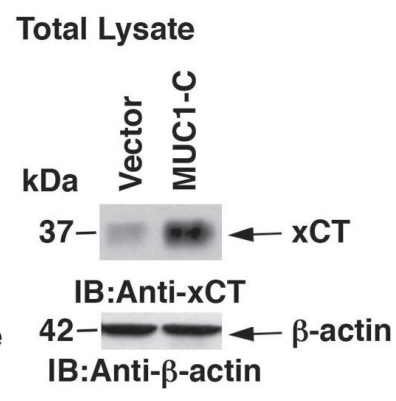

IB:Anti-MUC1-C

C. 293T

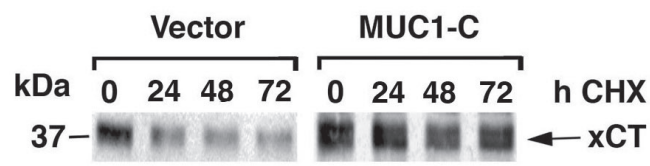

IB:Anti-xCT

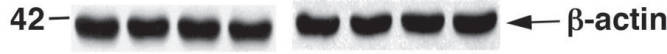

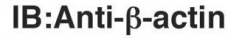

D. 293T

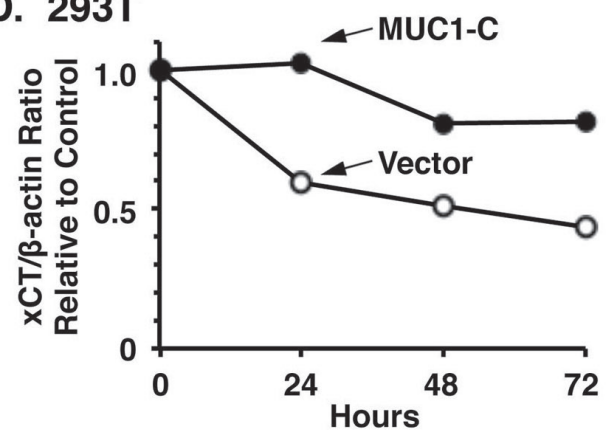

F. MDA-MB-468

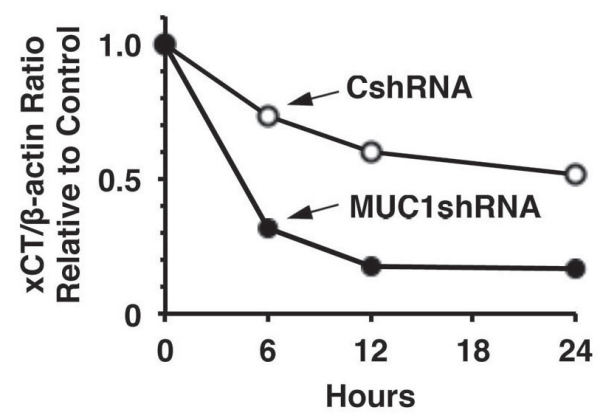

Figure 3: MUC1 increases xCT stability. A. 293T cells were transfected to stably express MUC1-C or a control vector. Lysates from the transduced cells were immunoblotted with the indicated antibodies (left). Lysates from 293T/MUC1-C cells were precipitated with anti-MUC1-C or a control IgG. The precipitates were immunoblotted with the indicated antibodies (right). B. Membrane fractions (left) and total cell lysates (right) from 293T/vector or 293T/MUC1-C cells were immunoblotted with the indicated antibodies. C. 293T/ vector and 293T/MUC1-C cells were exposed to $50 \mu \mathrm{g} / \mathrm{ml} \mathrm{CHX}$ for the indicated times. Total cell lysates were immunoblotted with the indicated antibodies. D. Intensities of the xCT signals as compared to those obtained for $\beta$-actin (xCT/ $\beta$-actin ratio) for the CHX-treated 293T/vector and 293T/MUC1-C cells are plotted relative to the control (time 0; assigned a value of 1). E. MDA-MB-468/CshRNA and MDA-MB-468/MUC1shRNA cells were exposed to CHX $(50 \mu \mathrm{g} / \mathrm{ml})$ for the indicated times. Total cell lysates were immunoblotted with the indicated antibodies. F. Intensities of the xCT signals as compared to those obtained for $\beta$-actin (xCT/ $\beta$-actin ratio) for the $\mathrm{CHX}$-treated MDA-MB-468/CshRNA and MDA-MB-468/MUC1shRNA cells are plotted relative to the control (time 0; assigned a value of 1). 
20 (Supplementary Figure S4B) cells stably expressing a second $\mathrm{xCT}$ shRNA (xCTshRNA-2), indicating that silencing $\mathrm{xCT}$ decreases MUC1-C expression.

Glutamate promotes the downregulation of MUC1-C expression

As expected, inhibiting $\mathrm{xCT}$ with SASP was associated with significant increases in intracellular glutamate levels (Supplementary Figure S5A). Based on these results, we treated MDA-MB-468 cells with glutamate and found downregulation of MUC1-C mRNA and protein (Figure 5A, left and right). Similar results were obtained with BT-20 cells (Figure 5B, left and right). Consistent with these findings, treatment with monosodium glutamate (MSG) similarly resulted in suppression of MUC1-C expression (Supplementary Figures S5B and S5C). The observation that glutamate suppresses MUC1-C expression prompted further studies on the effects of inhibiting the conversion of glutamine to glutamate by glutaminases (GLSs) [25]. Indeed, treatment of MDA-MB-468 cells with the GLS inhibitor Compound 968 was associated with upregulation of MUC1-C mRNA
A. MDA-MB-468

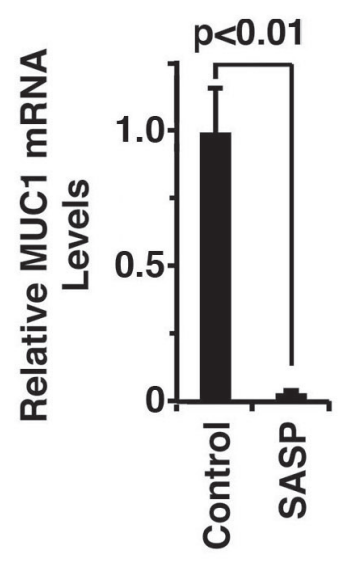

C. MDA-MB-468

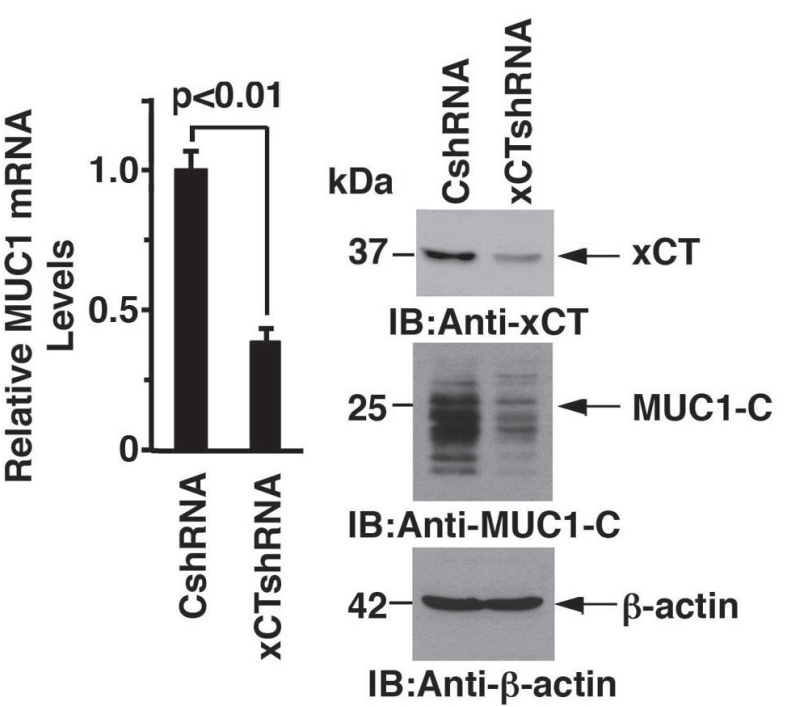

B. BT-20

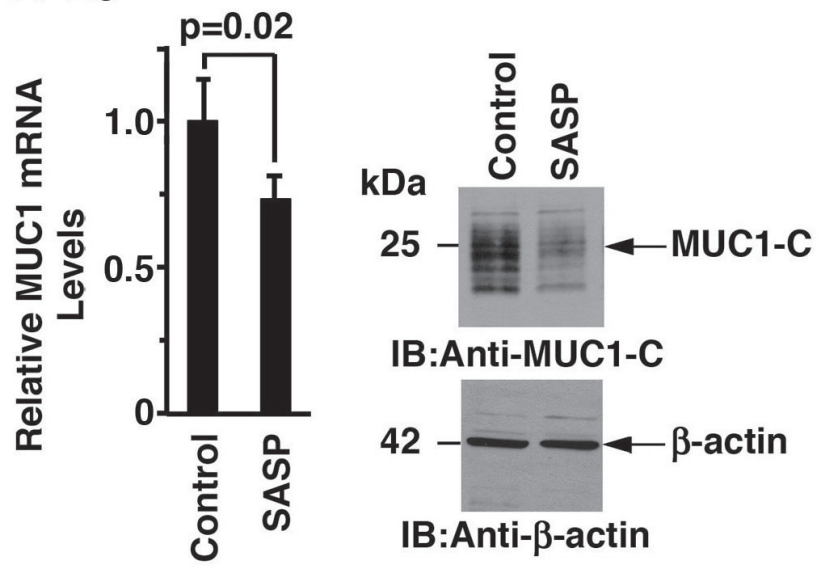

D. BT-20

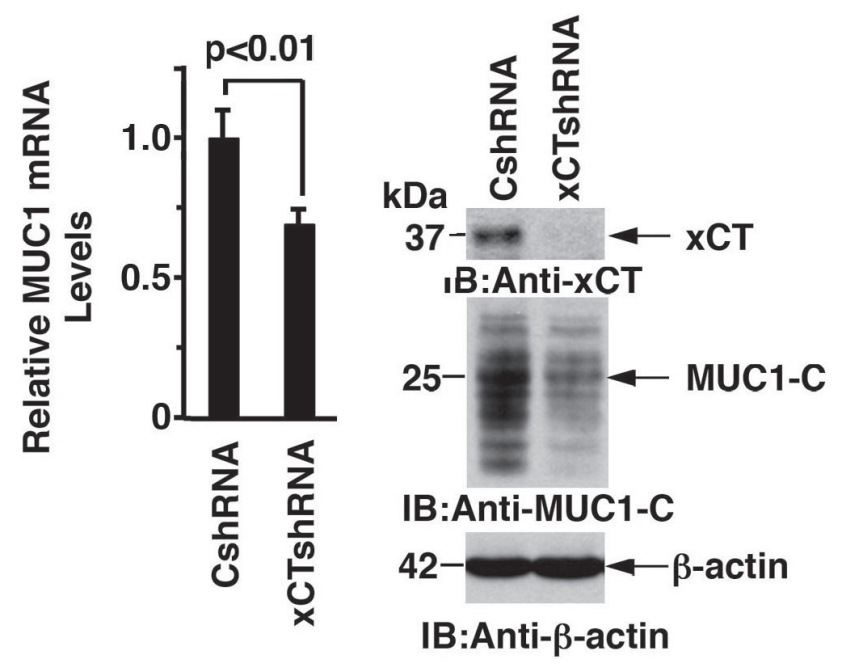

Figure 4: Targeting xCT suppresses MUC1 expression. A. and B. MDA-MB-468 (A) or BT-20 (B) cells were treated with $1.0 \mu \mathrm{M}$ or $1.5 \mu \mathrm{M}$ SASP for $72 \mathrm{~h}$, respectively. MUC1 mRNA levels were determined by qRT-PCR (left). The results (mean \pm SD of 4 determinations) are expressed as relative MUC1 mRNA levels as compared with that obtained for the untreated control cells (assigned a value of 1). Lysates from control and SASP-treated cells were immunoblotted with the indicated antibodies (right). C. and D. MDAMB-468 (C) and BT-20 (D) cells were transfected to stably express a control shRNA (CshRNA) or an xCT shRNA (xCTshRNA). MUC1 mRNA levels were determined by qRT-PCR (left). The results (mean \pm SD of 4 determinations) are expressed as relative MUC1 mRNA levels as compared with that obtained for the CshRNA cells (assigned a value of 1). Lysates were immunoblotted with the indicated antibodies (right). 
and protein (Figure 5C, left and right). BT-20 cells also responded to Compound 968 with increases in MUC1-C expression (Figure 5D, left and right), supporting a model in which MUC1-C expression is downregulated by targeting $\mathrm{xCT}$ and increasing glutamate levels.

\section{Role of $x C T$ in epigenetic modification of the} MUC1 promoter

$\mathrm{xCT}$ and $\mathrm{CD} 44 \mathrm{v}$ can function as a link to epigenetic regulatory mechanisms in cancer cells [26, 27]. Additionally, the epigenetic control of MUC1 in cancer cells has been associated with $\mathrm{H} 3 \mathrm{~K} 9$ modification and DNA methylation [19]. Accordingly, we investigated whether targeting $\mathrm{xCT}$ with SASP affects H3K9 methylation on the MUC1 promoter. Indeed, SASP treatment of MDA-MB-468 (Figure 6A, left and right) and BT-20 (Figure 6B, left and right) cells was associated with significant increases in both $\mathrm{H} 3 \mathrm{~K} 9 \mathrm{me} 2$ and $\mathrm{H} 3 \mathrm{~K} 9 \mathrm{me} 3$. In concert with these results, we also found that silencing xCT increases $\mathrm{H} 3 \mathrm{~K} 9$ methylation of the $M U C 1$ promoter in MDA-MB-468 (Figure 6C, right) and BT-20 (Figure $6 \mathrm{D}$, left and right) cells. In addition, $\mathrm{xCT}$ silencing was associated with marked increases in DNA methylation of the $M U C 1$ promoter (Figures 6E, left and right), consistent with the findings that targeting $\mathrm{xCT}$ downregulates MUC1-C expression.

\section{A. MDA-MB-468}

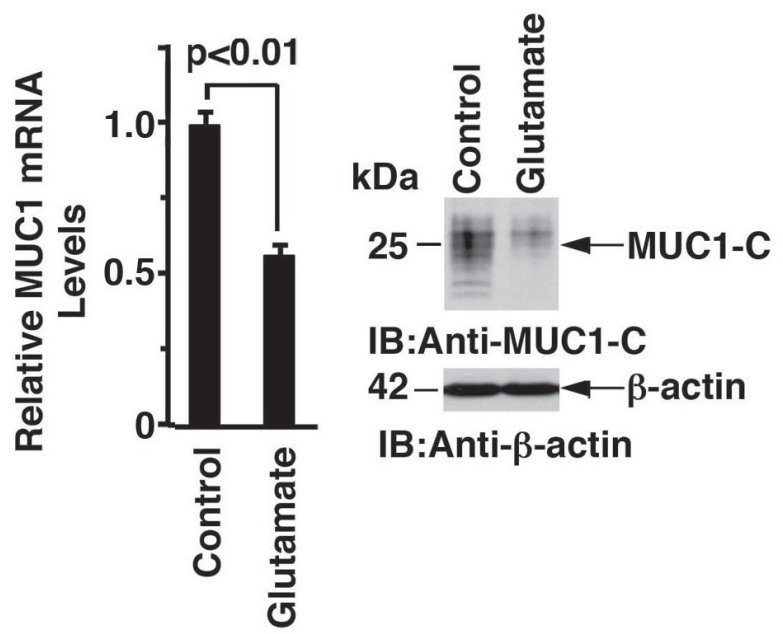

B. BT-20

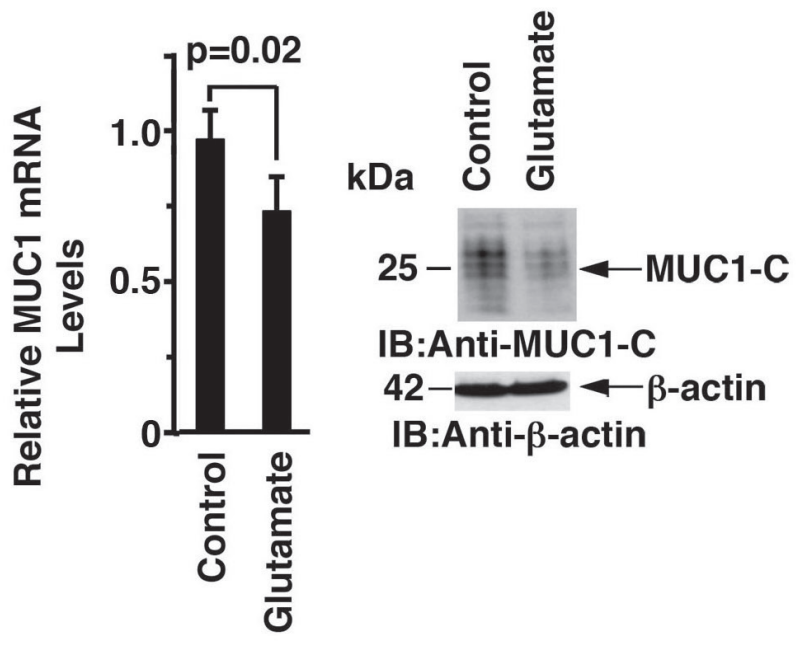

C. MDA-MB-468

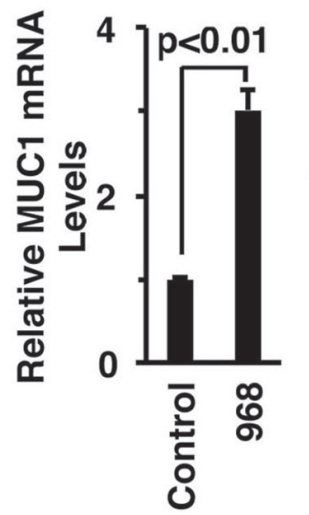

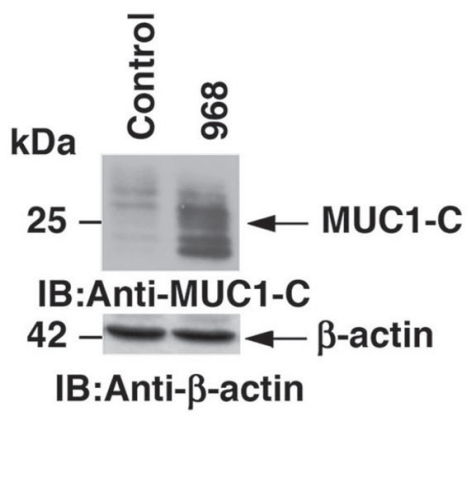

D. BT-20
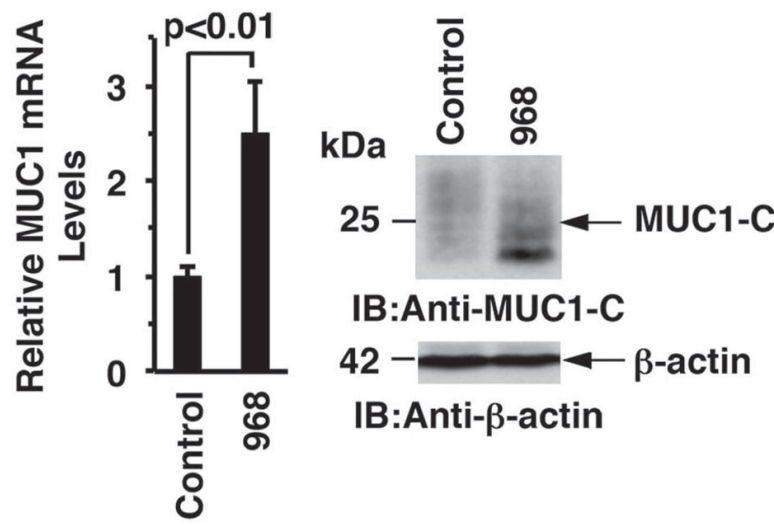

IB:Anti-MUC1-C

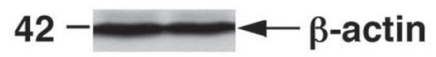

IB:Anti- $\beta$-actin

Figure 5: Glutamate suppresses MUC1 expression. A. and B.. MDA-MB-468 (A) or BT-20 (B) cells were treated with 20 mM glutamate for $72 \mathrm{~h}$. MUC1 mRNA levels in the control and glutamate treated cells were determined by qRT-PCR (left). The results (mean \pm SD of 4 determinations) are expressed as relative MUC1 mRNA levels as compared with that obtained for the untreated control cells (assigned a value of 1). Lysates were immunoblotted with the indicated antibodies (right). C. and D. MDA-MB-468 (C) or BT-20 (D) cells were treated with $10 \mu \mathrm{M}$ or $20 \mu \mathrm{M}$ compound 968 for $72 \mathrm{~h}$, respectively. MUC1 mRNA levels were determined by qRT-PCR (left). The results (mean $\pm \mathrm{SD}$ of 4 determinations) are expressed as relative MUC1 mRNA levels as compared with that obtained for the untreated control cells (assigned a value of 1). Lysates were immunoblotted with the indicated antibodies (right). 


\section{Targeting the MUC1-C/xCT pathway induces ferroptosis and decreases survival}

The small molecule erastin inhibits system $X_{C}$ mediated cystine uptake and induces an iron-dependent form of cell death known as ferroptosis [28]. Based on our findings that MUC1-C stabilizes $\mathrm{xCT}$, we asked if targeting MUC1-C affects erastin-induced ferroptosis. Treatment of MDA-MB-468 cells with erastin for $24 \mathrm{~h}$ had no effect on MUC1-C expression (Supplementary Figure S6A) or cell death (Figure 7A). By contrast, exposure to DOX for $7 \mathrm{~d}$ and then treatment with erastin was associated a marked induction of cell death in MDA-MB-468/tetMUC1shRNA, but not MDA-MB-468/tet-CshRNA, cells (Figure 7A). Moreover, we found that erastin-induced death of MDA-MB-468/tet-MUC1shRNA cells with
MUC1-C suppression was completely blocked by Fer-1, a lipid ROS scavenger that selectively inhibits ferroptosis [28](Figure 7A). In concert with the functional importance of MUC1-C, erastin-induced ferroptosis in DOX-treated MDA-MB-468/tet-MUC1shRNA cells was associated with significant decreases in GSH levels (Figure 7B). In addition, we found that BT-20/tet-MUC1shRNA, and not BT-20/tet-CshRNA, cells exposed to DOX were sensitive to erastin, confirming that MUC1-C blocks erastin-induced ferroptosis (Figure 7C and Supplementary Figure S6B) and induces increases in GSH (Figure 7D). These findings supported a model in which the MUC1-C/xCT signaling suppresses ferroptosis and thereby promotes survival of TNBC cells (Figure 7E).

\section{A. MDA-MB-468}

H3K9me2

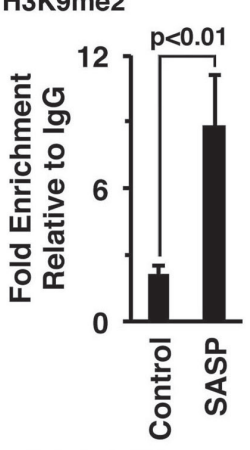

B. BT-20

H3K9me2

H3K9me3

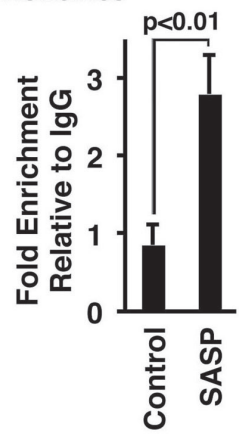

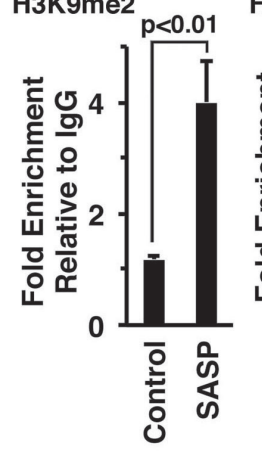

H3K9me3

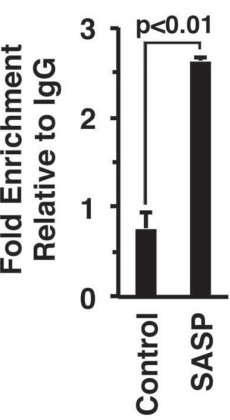

C. MDA-MB-468 H3K9me2 H3K9me3
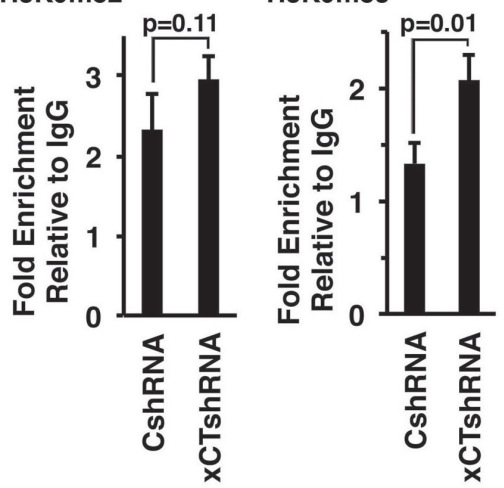

D. BT-20

H3K9me2

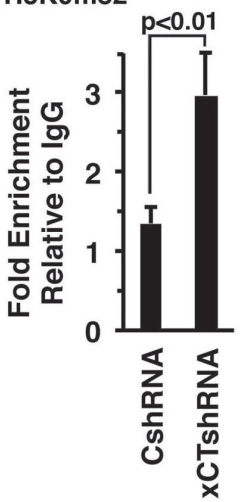

H3K9me3

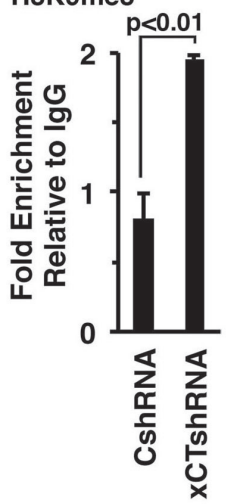

E.

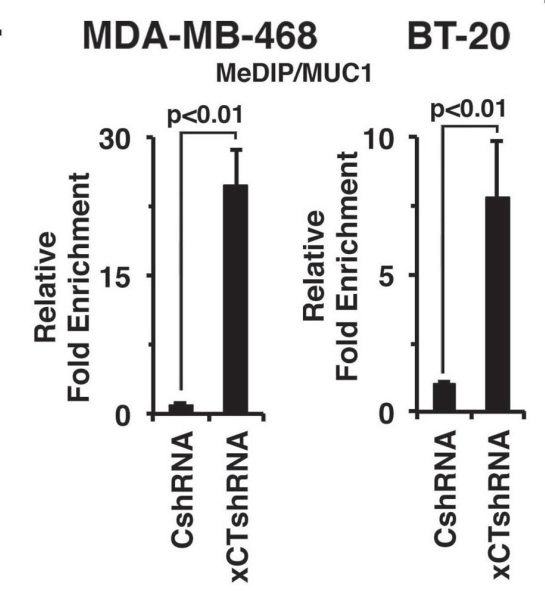

Figure 6: Targeting $\mathbf{x C T}$ induces histone and DNA methylation of the $\boldsymbol{M U C 1}$ promoter. A. and B. MDA-MB-468 (A) or BT20 (B) cells were treated with $1.0 \mathrm{mM}$ or $1.5 \mathrm{mM}$ SASP for $72 \mathrm{~h}$, respectively. Soluble chromatin from control and SASP treated cells was precipitated with anti-H3K9me2, anti-H3K9me3 or a control IgG. The final DNA samples were amplified by qPCR with pairs of primers for the $M U C 1$ promoter region or control region from the GAPDH promoter. The results (mean $\pm \mathrm{SD}$ of 3 determinations) are expressed as the relative fold enrichment compared with that obtained with the IgG control (untreated cells, assigned a value of 1). C. and D. Soluble chromatin from MDA-MB-468/CshRNA, MDA-MB-468/xCTshRNA (C), BT-20/CshRNA and BT-20/xCTshRNA (D) cells was precipitated with anti-H3K9me2, anti-H3K9me3 or a control IgG. The final DNA samples were amplified by qPCR with pairs of primers for the $M U C 1$ promoter region or control GAPDH promoter. The results (mean $\pm \mathrm{SD}$ of 3 determinations) are expressed as the relative fold enrichment compared with that obtained with the IgG control (CshRNA cells, assigned a value of 1). E. Genomic DNA from the indicated MDA-MB-468 (left) and BT-20 (right) cells was subjected to immunoprecipitation of methylated DNA (MeDIP) and the precipitates were analyzed by qPCR of the $M U C 1$ gene promoter. The results (mean $\pm \mathrm{SD}$ of 3 determinations) are expressed as relative fold enrichment compared to that obtained from CshRNA expressing cells (assigned a value of 1). 

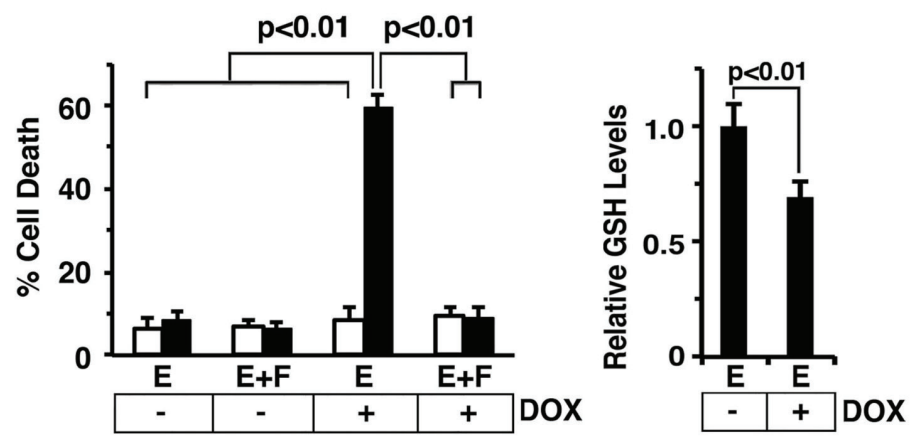

C. BT-20

D. BT-20
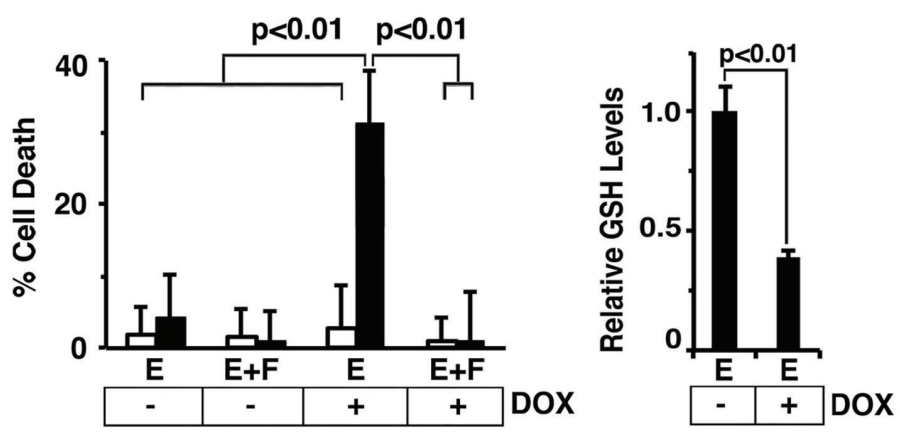

E.

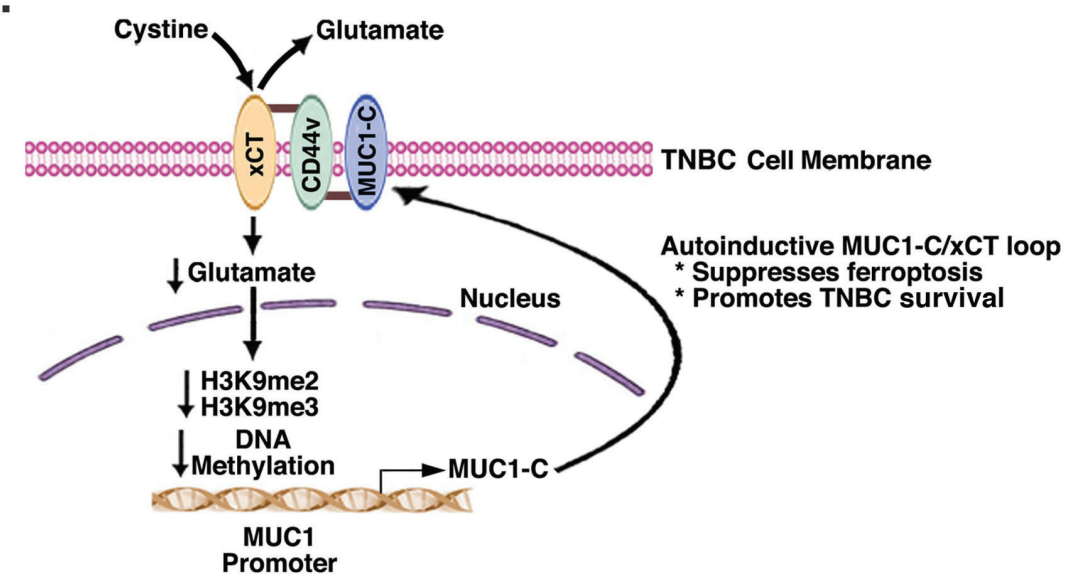

Figure 7: MUC1-C protects against erastin-induced ferroptosis. A. and B. MDA-MB-468/tet-CshRNA (open bars) and MDAMB-468/tet-MUC1shRNA (solid bars) cells were treated with $200 \mathrm{ng} / \mathrm{ml} \mathrm{DOX} \mathrm{for} 7 \mathrm{~d}$. A. Cells were then plated in a 96 well plate, and exposed to $1 \mu \mathrm{M}$ erastin (labeled E) with or without $2 \mu \mathrm{M}$ Fer-1 (labeled F) for $24 \mathrm{~h}$. The results (mean \pm SD of 8 determinations) are expressed as percentage cell death as determined by Alamar blue analysis. B. The indicated cells were analyzed for relative GSH levels (mean $\pm \mathrm{SD}$ of 3 determinations) as compared with that obtained for erastin-treated, DOX- cells (assigned a value of 1)(right). C. and D. BT-20/tet-CshRNA (open bars) and BT-20/tet-MUC1shRNA (solid bars) cells were treated with $200 \mathrm{ng} / \mathrm{ml} \mathrm{DOX} \mathrm{for} 7$ d. C. Cells were then plated in a 96 well plate, and exposed to $0.5 \mu \mathrm{M}$ erastin (labeled E) with or without $2 \mu \mathrm{M}$ Fer-1 (labeled F) for $12 \mathrm{~h}$. The results (mean $\pm \mathrm{SD}$ of 8 determinations) are expressed as percentage cell death as determined by Alamar blue analysis. D. The indicated cells were analyzed for relative GSH levels (mean \pm SD of 3 determinations) as compared with that obtained for erastin-treated, DOX- cells (assigned a value of 1)(right). E. Schema of the proposed MUC1-C/xCT regulatory loop. MUC1-C forms a complex with $\mathrm{xCT}$ and $\mathrm{CD} 44 \mathrm{v}$ in the TNBC cell membrane through a direct interaction between the MUC1-C cytoplasmic domain (MUC1-CD) and the CD44v intracellular domain (CD44v-ICD). CD44v interacts with xCT through their extracellular domains [7]. MUC1-C stabilizes xCT; thus targeting MUC1-C decreases $\mathrm{xCT}$ in the cell membrane. In addition, targeting $\mathrm{xCT}$ with silencing or SASP increases intracellular glutamate and thereby suppresses MUC1 transcription by increasing H3K9 and DNA methylation on the MUC1 promoter. The MUC1-C/xCT loop suppresses ferroptosis and promotes TNBC cell survival. 


\section{DISCUSSION}

The MUC1-C transmembrane protein is expressed on the apical borders of normal epithelial cells [23]. However, with transformation and loss of polarity, MUC1-C is upregulated and repositioned over the entire cell membrane, where it forms complexes with cell surface receptors normally positioned at the basolateral borders [12]. The present studies demonstrate that MUC1-C associates with the $\mathrm{xCT}$ transporter at the cell membrane. Notably, like MUC1-C [12, 13, 23], xCT is highly expressed in diverse malignancies, including TNBC, and is induced by chemotherapy [4-6, 8, 9]. $\mathrm{CD} 44 \mathrm{v}$ forms a complex with $\mathrm{xCT}$ and in turn stabilizes $\mathrm{xCT}$ in gastrointestinal cancer cells [7]. In this respect, our results further indicate that MUC1-C interacts with the $\mathrm{xCT} / \mathrm{CD} 44 \mathrm{v}$ complex (Figure 7E). The MUC1-C cytoplasmic domain is an intrinsically disordered protein [22], that like other oncogenic molecules in this class [29], has the capacity to interact with diverse effectors and direct the activation of multiple signaling pathways [12]. In concert with this potential, our binding studies demonstrate that the MUC1-C cytoplasmic domain interacts directly with the CD44 intracellular domain (ICD). Expression of CD44 and particularly the variant $\mathrm{CD} 44 \mathrm{v}$ isoforms is upregulated in carcinomas and, as an adhesion molecule, plays a role in tumor cell invasion and metastases [7, 30, 31]. CD44v also functions in regulating redox balance in CSCs [26], at least in part by interacting with the $\mathrm{xCT}$ extracellular region and thereby stabilizing $\mathrm{xCT}$ [7]. In this way, our results support a model in which (i) MUC1-C binds intracellularly to the CD44-ICD, (ii) CD44 interacts extracellularly with $\mathrm{xCT}$, and in turn, (iii) MUC1-C promotes the stabilization of $\mathrm{xCT}$ (Figure 7E). Of note, these findings do not exclude the possibility that MUC1-C can also (i) bind to and directly stabilize $\mathrm{xCT}$, or (ii) interact with the system $X_{C}^{-} 4 F 2$ heavy chain; however, further studies will be needed to address these issues.

MUC1-C is aberrantly overexpressed in breast carcinomas by mechanisms involving in part genetic alterations and dysregulation of transcription [12]. Surprisingly, however, little is known about epigenetic regulation of the $M U C 1$ gene in cancer cells [19]. In the present studies, we made the unexpected finding that targeting $\mathrm{xCT}$ is associated with the downregulation of MUC1 transcription. Thus, treatment of TNBC cells with the $\mathrm{xCT}$ inhibitor SASP decreased MUC1 mRNA and MUC1-C protein. Moreover, silencing $\mathrm{xCT}$ with different shRNAs similarly resulted in downregulation of MUC1-C expression, providing initial support for a MUC1-C/ $\mathrm{xCT}$ positive regulatory loop (Figure 7E). As expected, targeting $\mathrm{xCT}$ was associated with increases in glutamate as a result of suppressing the cystine/glutamate transporter function. Accordingly, we found that exposure of TNBC cells to glutamate suppressed MUC1-C expression, invoking the possibility that the glutamate metabolic pathway drives epigenetic downregulation of the $M U C 1$ promoter. Activation of the $M U C 1$ promoter has been linked to autoinductive loops involving the (i) NF- $\mathrm{kB}$ p65 $[32,33]$, and (ii) STAT1/3 [18, 34] signaling. However, to our knowledge, there are no reports demonstrating a link between $\mathrm{xCT}$ metabolic pathways and epigenetic regulation of $M U C 1$ promoter. In this context and based on the potential importance of H3K9 modification on the $M U C 1$ promoter [19], we found that targeting $\mathrm{xCT}$ is associated with significant increases in $\mathrm{H} 3 \mathrm{~K} 9 \mathrm{me} 2$ and H3K9me3 occupancy, supporting a mechanism for suppression of $M U C 1$ transcription (Figure 7E). In addition, the $\mathrm{MUCl}$ promoter includes multiple $\mathrm{CpG}$ sites that contribute to the regulation of MUC1 expression in response to 5-aza-2'-deoxycytidine [19]. Notably, xCT targeting was also associated with marked induction of DNA methylation on the $M U C 1$ promoter (Figure 7E). These results thus support the premise that $\mathrm{xCT}$ suppresses H3K9 and DNA methylation of the MUC1 promoter and thereby promotes MUC1-C expression (Figure 7E).

Targeting MUC1-C in breast and other cancer cells is associated with decreases in GSH, disruption of redox balance and ROS-mediated death by mechanisms that have remained unclear $[12,17,35,36]$. In this respect, stabilization of $\mathrm{xCT}$ by CD44v in cancer cells increases intracellular cystine and enhances the capacity for GSH synthesis [7]. Thus, the present findings that targeting MUC1-C decreases XCT stability lend credence to the notion that MUC1-C maintains redox balance by promoting a positive regulatory loop with the $\mathrm{xCT}$ pathway (Figure 7E). Implicit in this model is that targeting MUC1-C decreases $\mathrm{xCT}$ and thereby induces ROS-mediated death. Based on these premises, we treated TNBC cells with erastin, an inhibitor of cystine uptake by system $\mathrm{X}_{\mathrm{C}}{ }^{-}$and inducer of ferroptosis, an iron-dependent form of nonapoptotic ROS-mediated cell death [28]. Importantly, erastin was ineffective in inducing ferroptosis in the presence of MUC1-C expression. By contrast, silencing MUC1-C was necessary for erastin-induced ferroptosis, which was substantiated by the suppression of cell death with Fer-1. Our findings therefore indicate that targeting of the MUC1-C/xCT signaling pathway represents a potential therapeutic approach to induce ferroptosis and thereby contribute to inhibition of MUC1-C-mediated TNBC cell self-renewal capacity and tumorigenicity [24] (Figure 7E). These findings of functional interactions between $\mathrm{xCT}, \mathrm{CD} 44 \mathrm{v}$ and MUC1-C in TNBC cells may also be more broadly applicable to other types of carcinomas. 


\section{MATERIALS AND METHODS}

\section{Cell culture}

MDA-MB-468 and MCF-7 breast cancer cells, and 293 T embryonic kidney cells were grown in DMEM media supplemented with $10 \%$ heat-inactivated FBS (HI-FBS), $100 \mu \mathrm{g} / \mathrm{ml}$ streptomycin, and 100 units $/ \mathrm{ml}$ penicillin. BT-20 breast cancer cells were grown in EMEM media supplemented with HI-FBS and antibiotics. ZR-75-1 breast cancer cells were grown in RPMI1640 media containing with HI-FBS and antibiotics. MDA-MB-468 and BT-20 cells were infected with a lentiviral vector expressing a MUC1shRNA (Sigma; TRCN0000122938), xCTshRNAs (Sigma; TRCN0000043126, TRCN0000043123) or with a scrambled control shRNA vector (CshRNA; Sigma) as described [37]. $293 \mathrm{~T}$ cells were transfected to stably express a control pIRES-puro2 or a pIRES-puro2MUC1-C vector [20]. Cells were treated with doxycycline (DOX), sulfasalazine (SASP), L-glutamate, monosodium glutamate (MSG), Compound 968 (Millipore), erastin (Selleckchem) or ferrostatin 1 (Fer-1) (Tocris Bioscience). Cell viability was determined using the Alamar blue assay as described [38].

\section{Immunoprecipitation and immunoblotting}

Total cell lysates were prepared in lysis buffer as described [36]. Membrane fractions were extracted from whole cell lysates using the Membrane Protein Extraction Kit (BioVision). Soluble proteins were subjected to immunoprecipitation with anti-MUC1-C [39] or anti-xCT (Novus). Precipitates and cell lysates were analyzed by immunoblotting with anti-MUC1-C, anti-xCT anti- $\beta$-actin (Sigma), anti-Na,K ATPase and anti-CD44 (Cell Signaling Technology). Immune complexes were detected using horseradish peroxidase-conjugated secondary antibodies and enhanced chemiluminescence (PerkinElmer). Quantification of signal intensity was performed using Image J software.

\section{Tetracycline-inducible MUC1 silencing}

A MUC1shRNA (MISSION shRNA; Sigma, TRCN0000122938) was cloned into the pLKO-tet-puro vector (Addgene, Plasmid \#21915). The pLKO-tet-puro vector was co-transfected with the lentivirus packaging plasmids into $293 \mathrm{~T}$ cells, and the supernatant containing the viral particles was collected at $48 \mathrm{~h}$ after transfection. MDA-MB-468 or BT-20 cells were incubated with the supernatant for $12 \mathrm{~h}$ in the presence of $8 \mu \mathrm{g} / \mathrm{ml}$ polybrene, followed by replacement with complete cell culture medium and selection in $1-3 \mu \mathrm{g} / \mathrm{ml}$ puromycin.

\section{Measurement of glutamate levels}

Intracellular glutamate levels were measured using the Glutamate Assay Kit (Abcam).

\section{Transient overexpression of CD44v}

CD44v8-10 was generated by RT-PCR using MDA-MB-468 total RNA as a template. The PCR products were digested with EcoRI/XhoI and then cloned into corresponding sites in pcDNA3. 293T cells were transfected with pcDNA3 or pcDNA3-CD44v with SuperFect Transfection Reagent (QIAGEN), and the cells were harvested at $48 \mathrm{~h}$ after transfection.

\section{In vitro binding assays}

GST, GST-MUC1-CD, GST-MUC1-CD(1-45), GST-MUC1-CD(46-72) GST-MUC1-CD(20-72) and GST-MUC1-CD(AQA) were prepared as described [18, 40]. The CD44 intracellular domain (CD44-ICD) was generated by RT-PCR using SK-CO-1 cell total RNA as a template. The PCR products were digested with EcoRI/ XhoI and then cloned into corresponding sites in pGEX5X-1 (GE Healthcare) to generate GST fusion proteins. Site-directed mutagenesis PCR (Agilent) was performed to generate deletion mutants of CD44-ICD. CD44-ICD PCR products were also digested with EcoRI/XhoI and cloned into corresponding sites in $\mathrm{pET}-28 \mathrm{~b}$ to generate His-tagged proteins. Purified GST-MUC1-CD was cleaved with thrombin to remove the GST moiety. For bindings assays, purified proteins were incubated for $2 \mathrm{~h}$ at room temperature. Adsorbates to glutathione-conjugated beads were analyzed by immunoblotting.

\section{Quantitative RT-PCR}

cDNA synthesis was performed with $2 \mu \mathrm{g}$ of total RNA using the SuperScript III First-Strand Synthesis System (Invitrogen). cDNA samples were then amplified using The Power SYBR Green PCR Master Mix (Applied Biosystems) and the 7300 Realtime PCR System (Applied Biosystems)[41]. Primers used for qRT-PCR analysis are included in Supplementary Table S1.

\section{Chromatin immunoprecipitation (ChIP) assays}

Soluble chromatin was prepared from cells as described [42] and precipitated with anti-trimethyl H3K9, anti-dimethyl H3K9 (Abcam) or a control nonimmune IgG. The SYBR green qPCR kit was used for ChIP qPCRs with the ABI Prism 7000 Sequence Detector (Applied Biosystems). Relative fold enrichment was calculated 
as described [43]. Primers used for qPCR of the MUC1 promoter and control region are listed in Supplementary Table S2.

\section{Methylated DNA immunoprecipitation (MeDIP) assays}

DNA methylation analysis was performed using the Methylation DNA IP (MeDIP) kit (Active Motif) by immunoprecipitating and enriching for DNA fragments containing 5-mC. Genomic DNA from CshRNA or xCTshRNA expressing cells was digested with Mse-1, heat denatured, and methylated DNA was precipitated with an anti-5-mC antibody (Active Motif). The precipitated DNA was subjected to qPCR analysis using the SYBR green master mix and amplification in an AB7000 sequence detector (Applied Biosystems). Primer sets used to analyze the MUC1 promoter are listed in Supplementary Table S2.

\section{Measurement of GSH levels}

Intracellular GSH concentrations were determined using the Bioxytech GSH-400 kit (OXIS International) as described.

\section{Abbreviations}

$\mathrm{xCT}$, Xc- light chain; TNBC, triple-negative breast cancer; CD44v, CD44 variant; CD44-ICD, CD44 intracellular domain; CSCs, cancer stem-like cells; MUC1, mucin 1; DOX, doxycycline; SASP, sulfasalazine; MSG, monosodium glutamate; GSH, glutathione; ChIP, chromatin immunoprecipitation; MeDIP, methylated DNA immunoprecipitation; CHX, cyclohexamide; Fer-1, ferrostatin 1

\section{Author contributions}

Conceptualization, M.H., H.T., V.P.S. and D.K.; Methodology, Y.S., V.P.S; Investigation, M.H., H.T., H.R., M.A., Y.S., L.Y., A.T., T.M. and M.H.; Writing-Original Draft, D.K.; Writing-Review \& Editing, M.H., H.T., V.P.S. and D.K.; Supervision, M.H., H.R., V.P.S. and D.K.; Funding Acquisition, V.P.S. and D.K.

\section{FINANCIAL SUPPORT}

Research reported in this paper was supported by the National Cancer Institute of the National Institutes of Health under award numbers CA097098 and CA166480, and by the Lung Cancer Research Foundation.

\section{CONFLICTS OF INTEREST}

D.K. holds equity in Genus Oncology and is a consultant to the company. The other authors disclosed no potential conflicts of interest.

\section{REFERENCES}

1. Lewerenz J, Hewett SJ, Huang Y, Lambros M, Gout PW, Kalivas PW, Massie A, Smolders I, Methner A, Pergande M, Smith SB, Ganapathy V and Maher P. The cystine/ glutamate antiporter system Xc- in health and disease: from molecular mechanisms to novel therapeutic opportunities. Antioxid Redox Signal. 2013; 18:522-555.

2. Tagde A, Singh H, Kang MH and Reynolds CP. The glutathione synthesis inhibitor buthionine sulfoximine synergistically enhanced melphalan activity against preclinical models of multiple myeloma. Blood Cancer J. 2014; 4:e229.

3. Sato H, Shiiya A, Kimata M, Maebara K, Tamba M, Sakakura Y, Makino N, Sugiyama F, Yagami K, Moriguchi $\mathrm{T}$, Takahashi S and Bannai S. Redox imbalance in cystine/ glutamate transporter-deficient mice. J Biol Chem. 2005; 280:37423-37429.

4. Huang L, Chen D, Liu D, Yin L, Kharbanda S and Kufe D. MUC1 oncoprotein blocks GSK3 $\beta$-mediated phosphorylation and degradation of $\beta$-catenin. Cancer Res. 2005; 65:10413-10422.

5. Lo M, Wang YZ and Gout PW. The Xc- cystine/glutamate antiporter: a potential target for therapy of cancer and other diseases. J Cell Physiol. 2008; 215:593-602.

6. Chen RS, Song YM, Zhou ZY, Tong T, Li Y, Fu M, Guo XL, Dong LJ, He X, Qiao HX, Zhan QM and Li W. Disruption of $\mathrm{xCT}$ inhibits cancer cell metastasis via the caveolin-1/beta-catenin pathway. Oncogene. 2009; 28:599609 .

7. Ishimoto T, Nagano O, Yae T, Tamada M, Motohara T, Oshima H, Oshima M, Ikeda T, Asaba R, Yagi H, Masuko T, Shimizu T, Ishikawa T, Kai K, Takahashi E, Imamura $\mathrm{Y}$, et al. CD44 variant regulates redox status in cancer cells by stabilizing the $\mathrm{xCT}$ subunit of system Xc-and thereby promotes tumor growth. Cancer Cell. 2011; 19:387-400.

8. Timmerman LA, Holton T, Yuneva M, Louie RJ, Padro M, Daemen A, Hu M, Chan DA, Ethier SP, van 't Veer LJ, Polyak K, McCormick F and Gray JW. Glutamine sensitivity analysis identifies the $\mathrm{xCT}$ antiporter as a common triple-negative breast tumor therapeutic target. Cancer Cell. 2013; 24:450-465.

9. Lu H, Samanta D, Xiang L, Zhang H, Hu H, Chen I, Bullen JW and Semenza GL. Chemotherapy triggers HIF-1dependent glutathione synthesis and copper chelation that induces the breast cancer stem cell phenotype. Proc Natl Acad Sci USA. 2015; 112:E4600-9.

10. Okuno S, Sato H, Kuriyama-Matsumura K, Tamba M, 
Wang H, Sohda S, Hamada H, Yoshikawa H, Kondo T and Bannai S. Role of cystine transport in intracellular glutathione level and cisplatin resistance in human ovarian cancer cell lines. Br J Cancer. 2003; 88:951-956.

11. Narang VS, Pauletti GM, Gout PW, Buckley DJ and Buckley AR. Sulfasalazine-induced reduction of glutathione levels in breast cancer cells: enhancement of growthinhibitory activity of Doxorubicin. Chemotherapy. 2007; 53:210-217.

12. Kufe D. MUC1-C oncoprotein as a target in breast cancer: activation of signaling pathways and therapeutic approaches. Oncogene. 2013; 32:1073-1081.

13. Siroy A, Abdul-Karim FW, Miedler J, Fong N, Fu $\mathrm{P}$, Gilmore $\mathrm{H}$ and Baar J. MUC1 is expressed at high frequency in early-stage basal-like triple-negative breast cancer. Hum Pathol. 2013; 44:2159-2166.

14. Bieche I and Lidereau R. A gene dosage effect is responsible for high overexpression of the MUC1 gene observed in human breast tumors. Cancer Genet Cytogenet. 1997; 98:75-80

15. Lacunza E, Baudis M, Colussi AG, Segal-Eiras A, Croce MV and Abba MC. MUC1 oncogene amplification correlates with protein overexpression in invasive breast carcinoma cells. Cancer Genet Cytogenet. 2010; 201:102110.

16. Khodarev N, Pitroda S, Beckett M, MacDermed D, Huang L, Kufe D and Weichselbaum R. MUC1-induced transcriptional programs associated with tumorigenesis predict outcome in breast and lung cancer. Cancer Res. 2009; 69:2833-2837.

17. Raina D, Ahmad R, Joshi M, Yin L, Wu Z, Kawano T, Vasir B, Avigan D, Kharbanda S and Kufe D. Direct targeting of the MUC1 oncoprotein blocks survival and tumorigenicity of human breast carcinoma cells. Cancer Res. 2009; 69:5133-5141.

18. Ahmad R, Rajabi H, Kosugi M, Joshi M, Alam M, Vasir B, Kawano T, Kharbanda S and Kufe D. MUC1-C oncoprotein promotes STAT3 activation in an auto-inductive regulatory loop. Sci Signal. 2011; 4:ra9.

19. Yamada N, Nishida Y, Tsutsumida H, Hamada T, Goto M, Higashi M, Nomoto M and Yonezawa S. MUC1 expression is regulated by DNA methylation and histone $\mathrm{H} 3$ lysine 9 modification in cancer cells. Cancer Res. 2008; 68:27082716.

20. Ramasamy S, Duraisamy S, Barbashov S, Kawano T, Kharbanda S and Kufe D. The MUC1 and galectin-3 oncoproteins function in a microRNA-dependent regulatory loop. Mol Cell. 2007; 27:992-1004.

21. Raina D, Uchida Y, Kharbanda A, Rajabi H, Panchamoorthy G, Jin C, Kharbanda S, Scaltriti M, Baselga J and Kufe D. Targeting the MUC1-C oncoprotein downregulates HER2 activation and abrogates trastuzumab resistance in breast cancer cells. Oncogene. 2014; 33:3422-3431.

22. Raina D, Agarwal P, Lee J, Bharti A, McKnight C, Sharma
P, Kharbanda $\mathrm{S}$ and Kufe D. Characterization of the MUC1-C cytoplasmic domain as a cancer target. PLoS One. 2015; 10:e0135156.

23. Kufe D. Mucins in cancer: function, prognosis and therapy. Nature Reviews Cancer. 2009; 9:874-885.

24. Alam M, Rajabi H, Ahmad R, Jin C and Kufe D. Targeting the MUC1-C oncoprotein inhibits self-renewal capacity of breast cancer cells. Oncotarget. 2014; 5:2622-2634. doi: 10.18632/oncotarget.1848.

25. Hensley CT, Wasti AT and DeBerardinis RJ. Glutamine and cancer: cell biology, physiology, and clinical opportunities. J Clin Invest. 2013; 123:3678-3684.

26. Nagano O, Okazaki S and Saya H. Redox regulation in stem-like cancer cells by CD44 variant isoforms. Oncogene. 2013; 32:5191-5198.

27. Hitchler MJ and Domann FE. Metabolic defects provide a spark for the epigenetic switch in cancer. Free Radic Biol Med. 2009; 47:115-127.

28. Dixon SJ, Lemberg KM, Lamprecht MR, Skouta R, Zaitsev EM, Gleason CE, Patel DN, Bauer AJ, Cantley AM, Yang WS, Morrison B, 3rd and Stockwell BR. Ferroptosis: an iron-dependent form of nonapoptotic cell death. Cell. 2012; 149:1060-1072.

29. Dyson HJ and Wright PE. Intrinsically unstructured proteins and their functions. Nat Rev Mol Cell Biol. 2005; 6:197208.

30. Tanabe KK, Ellis LM and Saya H. Expression of CD44R1 adhesion molecule in colon carcinomas and metastases. Lancet. 1993; 341:725-726.

31. Yan Y, Zuo X and Wei D. Concise Review: Emerging role of CD44 in cancer stem cells: A promising biomarker and therapeutic target. Stem cells translational medicine. 2015; 4:1033-1043.

32. Ahmad R, Raina D, Trivedi V, Ren J, Rajabi H, Kharbanda

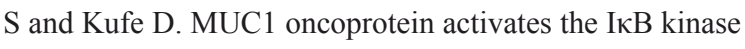
$\beta$ complex and constitutive NF- $\kappa \mathrm{B}$ signaling. Nat Cell Biol. 2007; 9:1419-1427.

33. Ahmad R, Raina D, Joshi MD, Kawano T, Kharbanda S and Kufe D. MUC1-C oncoprotein functions as a direct activator of the NF- $\kappa \mathrm{B}$ p 65 transcription factor. Cancer Res. 2009; 69:7013-7021.

34. Khodarev N, Ahmad R, Rajabi H, Pitroda S, Kufe T, McClary C, Joshi MD, MacDermed D, Weichselbaum R and Kufe D. Cooperativity of the MUC1 oncoprotein and STAT1 pathway in poor prognosis human breast cancer. Oncogene. 2010; 29:920-929.

35. Joshi MD, Ahmad R, Raina D, Rajabi H, Bubley G, Kharbanda S and Kufe D. MUC1 oncoprotein is a druggable target in human prostate cancer cells. Mol Cancer Ther. 2009; 8:3056-3065.

36. Raina D, Kosugi M, Ahmad R, Panchamoorthy G, Rajabi H, Alam M, Shimamura T, Shapiro G, Supko J, Kharbanda $\mathrm{S}$ and Kufe D. Dependence on the MUC1-C oncoprotein in non-small cell lung cancer cells. Mol Cancer Therapeutics. 
2011; 10:806-816.

37. Jin C, Rajabi H, Pitroda S, Kharbanda A, Li A, Weichselbaum R and Kufe D. Cooperative interaction between the MUC1 oncoprotein and the Rab31 GTPase in human breast cancer cells. PLoS One 2012; 7:e39432

38. Hasegawa M, Sinha RK, Kumar M, Alam M, Yin L, Raina D, Kharbanda A, Panchamoorthy G, Gupta D, Singh $\mathrm{H}$, Kharbanda $\mathrm{S}$ and Kufe D. Intracellular targeting of the oncogenic MUC1-C protein with a novel GO-203 nanoparticle formulation. Clin Cancer Res. 2015; 21:23382347.

39. Panchamoorthy G, Rehan H, Kharbanda A, Ahmad R and Kufe D. A monoclonal antibody against the oncogenic mucin 1 cytoplasmic domain. Hybridoma. 2011; 30:531535.

40. Rajabi H, Ahmad R, Jin C, Kosugi M, Alam M, Joshi M and Kufe D. MUC1-C oncoprotein induces TCF7L2 activation and promotes cyclin D1 expression in human breast cancer cells. J Biol Chem. 2012; 287:10703-10713.

41. Takahashi H, Jin C, Rajabi H, Pitroda S, Alam M, Ahmad R, Raina D, Hasegawa M, Suzuki Y, Tagde A, Bronson RT, Weichselbaum R and Kufe D. MUC1-C activates the TAK1 inflammatory pathway in colon cancer. Oncogene. 2015; 34:5187-97.

42. Alam M, Ahmad R, Rajabi H, Kharbanda A and Kufe D. MUC1-C oncoprotein activates ERK $\rightarrow \mathrm{C} / \mathrm{EBP} \beta$-mediated induction of aldehyde dehydrogenase activity in breast cancer cells. J Biol Chem. 2013; 288:30829-30903.

43. Wang Q, Carroll JS and Brown M. Spatial and temporal recruitment of androgen receptor and its coactivators involves chromosomal looping and polymerase tracking. Mol Cell. 2005; 19:631-642. 